\title{
Erratum to: Effectiveness of a home-based cognitive behavioral program to manage concerns about falls in community-dwelling, frail older people: results of a randomized controlled trial
}

Tanja A. C. Dorresteijn ${ }^{1 *}$, G. A. Rixt Zijlstra', Antonius W. Ambergen ${ }^{2}$, Kim Delbaere ${ }^{3}$, Johan W. S. Vlaeyen ${ }^{4,5}$ and Gertrudis I. J. M. Kempen ${ }^{1}$

\section{Erratum}

Unfortunately, the original version of this article [1] contains an error within Table 5 of the results section. Within the column "Intervention group" the number of "Indoor falls" was incorrectly written as 2, but should in fact be 202. The correct version of Table 5 can be found below.

\section{Author details \\ 'Department of Health Services Research - Focusing on Chronic Care and Ageing, CAPHRI School for Public Health and Primary Care, Maastricht University, P.O. Box 616, Maastricht, MD 6200, The Netherlands. ${ }^{2}$ Department of Methodology and Statistics, CAPHRI School for Public Health and Primary Care, Maastricht University, Maastricht, The Netherlands. ${ }^{3}$ Neuroscience Research Australia, University of New South Wales, Barker St, New South Wales, Australia. ${ }^{4}$ Research Group Health Psychology, University of Leuven, Leuven, Belgium. ${ }^{5}$ Department of Clinical Psychological Science, Maastricht University, Maastricht, The Netherlands.}

Received: 5 May 2016 Accepted: 5 May 2016

Published online: 24 May 2016

\section{Reference}

1. Dorresteijn TAC, Zijlstra GAR, Ambergen AW, Delbaere K, Vlaeyen JWS, Kempen GIJM. Effectiveness of a home-based cognitive behavioral program to manage concerns about falls in community-dwelling, frail older people: results of a randomized controlled trial. BMC Geriatr. 2016; 16:2. doi:10.1186/s12877-015-0177-y.

\footnotetext{
* Correspondence: t.dorresteijn@maastrichtuniversity.nl

'Department of Health Services Research - Focusing on Chronic Care and Ageing, CAPHRI School for Public Health and Primary Care, Maastricht University, P.O. Box 616, Maastricht, MD 6200, The Netherlands
}

\footnotetext{
Submit your next manuscript to BioMed Central and we will help you at every step:

- We accept pre-submission inquiries

- Our selector tool helps you to find the most relevant journal

- We provide round the clock customer support

- Convenient online submission

- Thorough peer review

- Inclusion in PubMed and all major indexing services

- Maximum visibility for your research
}

Submit your manuscript at

www.biomedcentral.com/submit

\section{C) Biomed Central}


Table 5 Effects of the Home-Based Cognitive Behavioral Program on Fall Outcomes

\begin{tabular}{|c|c|c|c|c|c|c|}
\hline & \multicolumn{2}{|c|}{ Control group } & \multicolumn{2}{|c|}{ Intervention group } & \multirow{3}{*}{$\begin{array}{l}\text { Model }^{a} \\
\text { OR (95\% Cl) }\end{array}$} & \multirow{3}{*}{$\begin{array}{l}P \text {-value } \\
P\end{array}$} \\
\hline & \multicolumn{2}{|c|}{$n=180$} & \multicolumn{2}{|c|}{$n=166$} & & \\
\hline & $n$ & (\%) & $n$ & (\%) & & \\
\hline \multicolumn{7}{|l|}{ Fallers } \\
\hline Baseline until 12-month follow-up & 106 & $(58.9)$ & 94 & $(56.6)$ & $0.79(0.50-1.23)$ & .292 \\
\hline \multicolumn{7}{|l|}{ Recurrent fallers } \\
\hline \multirow[t]{2}{*}{ Baseline until 12-month follow-up } & 67 & $(37.2)$ & 55 & $(33.1)$ & $0.67(0.41-1.09)$ & .104 \\
\hline & \multicolumn{2}{|c|}{ Number $^{b}$} & \multicolumn{2}{|c|}{ Number ${ }^{b}$} & IRR $(95 \% \mathrm{Cl})$ & P \\
\hline Total falls & \multicolumn{2}{|l|}{429} & \multicolumn{2}{|c|}{362} & $0.86(0.65-1.13)$ & .273 \\
\hline Indoor falls & \multicolumn{2}{|l|}{291} & \multicolumn{2}{|c|}{202} & $0.68(0.50-0.92)$ & .014 \\
\hline Outdoor & \multicolumn{2}{|l|}{138} & \multicolumn{2}{|c|}{160} & $1.11(0.78-1.56)$ & .568 \\
\hline No. of times medical attention required as a result of falls & \multicolumn{2}{|l|}{87} & \multicolumn{2}{|c|}{106} & $1.42(0.96-2.10)$ & .083 \\
\hline
\end{tabular}

Results of mixed-effects logistic and negative binomial regression analyses

$95 \% \mathrm{Cl}=95 \%$ confidence interval; $\mathrm{OR}=$ odds ratio mixed-effects logistics regression; IRR = incidence rate ratio obtained via negative binomial regression ${ }^{a}$ Model adjusted for baseline score measurement and level of concerns about falls, age, gender, perceived general health, and falls in the past 6 months

${ }^{\mathrm{b}}$ Analyses were performed with a Poisson distribution. This distribution of fall events accounts for over dispersion and incorporates both number of falls and time (weeks) of follow-up; herefore, all available data was used 